\title{
The Confidence Interval for the Periodic Intensity Function in the Presence of Power Function Trend on the Nonhomogeneous Poisson Process
}

\author{
Ikhsan Maulidi' ${ }^{1}$, Bonno Andri Wibowo ${ }^{2}$, Nina Valentika ${ }^{3}$, Muhammad Syazali $^{4}$, \\ Vina Apriliani ${ }^{5}$ \\ ${ }^{1}$ Department of Mathematics, Syiah Kuala University, Banda Aceh, Indonesia \\ ${ }^{2}$ Department of Mathematics, IPB University, Bogor, Indonesia \\ ${ }^{3}$ Department of Mathematics, Pamulang University, Tangerang Selatan, Indonesia \\ ${ }^{4}$ Department of Mathematics, Universitas Pertahanan, Bogor, Indonesia \\ ${ }^{5}$ Department of Mathematics Education, UIN Ar-Raniry, Banda Aceh, Indonesia
}

Email: ikhsanmaulidi@unsyiah.ac.id bonno1818@gmail.com , dosen02339@unpam.ac.id, muhamad.syazali@idu.ac.id, vina.apriliani@ar-raniry.ac.id

\begin{abstract}
The nonhomogeneous Poisson process is one of the most widely applied stochastic processes. In this article, we provide a confidence interval of the intensity estimator in the presence of a periodic multiplied by trend power function. This estimator's confidence interval is an application of the formulation of the estimator asymptotic distribution that has been given in previous studies. By using the asymptotic theorem, the distribution was derived in the form of a confidence interval for the intensity function. In addition, constructive proof of the convergent in probability has been provided for all power functions. The results of this study contribute to the study of statistical analysis of the estimators that have been formulated previously.
\end{abstract}

Keywords: asymptotic distribution; interval confidence; intensity function; Poisson process.

\section{INTRODUCTION}

There are many events in nature can be modeled by stochastic modeling processes. The stochastic process is a set of random variables that map the sample space to a state space. One of the stochastic processes is a counting process which states the number of events at a time interval. The counting process assuming the number of events has a Poisson distribution is called the Poisson process. Some basic theories related Poisson process can be seen in [1]-[3].

Due to the intensity function, the Poisson process is divided into two categories, namely the homogeneous Poisson process and the nonhomogeneous Poisson process. A homogeneous Poisson process has a constant intensity function (independent of time), while a nonhomogeneous Poisson process has a time-dependent intensity function. This nonhomogeneous Poisson process is widely applied to real phenomena, such as the phenomenon of earthquakes [4], traffic accidents [5], and radio burst rates [6].

On the other hand, the study of the nonhomogeneous Poisson process in the form a periodic intensity function also has been conducted in recent years. [7] studied the 
estimation of the intensity function in a nonhomogeneous Poisson process by including a trend component in the periodic intensity function. The trend component began with a trend in the form of an additive linear function. Then the study was continued with a trend in the form of a multiplicative linear function [8]. The estimation of the intensity function was carried out using the general kernel function approach, and [9] examined this Poisson process with a uniform kernel approach. Other related studies can be seen in [10]-[13].

[14] studied the estimation of the periodic Poisson process intensity function with the power function trend using a general kernel. Furthermore, the statistical properties of these estimators have also been proven. [15] has given strong consistency of these estimators. In addition, the asymptotic normality of the estimator has also been formulated and given a numerical simulation of the consistency of the estimator [16]. The results obtained in that study are the estimator of the periodic component which converges to the normal distribution by providing certain conditions.

As an application of the asymptotic normality, it can be determined the confidence interval of the estimator for the periodic component. This study provides the theorems for the confidence interval for the intensity function parameters and their proofs. The contribution of this study is to provide the characteristics of the estimator, especially in terms of accuracy. With a certain number of samples (interval length), it can be determined how accurately the estimator predicts the value of the parameter in the form of a confidence interval.

\section{METHODS}

\section{The Estimator for Periodic Component of the Intensity Function}

Suppose that $\{N(t), t \geq 0\}$ is a nonhomogeneous Poisson process with intensity function $\lambda$ which locally integrable and unknown. Suppose also that $\lambda$ is a periodic function with the trend of the power function, then $\lambda$ which depends on the time variable $s$ can be expressed as

$$
\lambda(s)=\lambda_{c}^{*}(s) \cdot a s^{b} .
$$

The values of the $a$ and $b$ constants are assumed to be known, so that what is not known is the function of the periodic component of the intensity function, namely $\lambda_{c}^{*}$. Equation (1) can also be stated as follows

$$
\lambda(s)=\lambda_{c}(s) . s^{b},
$$

with $\lambda_{c}(s)=a \lambda_{c}^{*}(s)$. [14] has been given the kernel type estimator for $\lambda_{c}(s)$ by using general kernel functions. The estimator for periodic component of the intensity is

$$
\hat{\lambda}_{c, n, k}(s)=\frac{\tau}{n} \sum_{k=0}^{\infty} \frac{1}{h_{n}(s+k \tau)^{b}} \int_{0}^{n} K\left(\frac{x-(s+k \tau)}{h_{n}}\right) N(d x) .
$$

On Equation (3), the constant $\tau$ is a period of the intensity function which satisfies

$$
\lambda_{c}(s+k \tau)=\lambda_{c}(s) \text {, for } k \in Z .
$$

With $n$ is the length of the time interval used. In this case, since the Poisson process is a discrete stochastic process, it is clear that $n$ is a natural number. The function $K$ called a kernel function if it satisfies the following properties: (K1) $K$ is a probability density function, (K2) $K$ is bounded, and (K3) $K$ is defined in [-1,1] [17]. 


\section{The Asymptotic Normality of the Estimator}

\section{Theorem 1 (The Asymptotic Normal Distribution for $\hat{\lambda}_{c, n, k}(s), 0<b<1$ )}

Suppose that the intensity $\lambda$ satisfies (1) and locally integrable. The kernel function $K$ satisfies (K1), (K2), (K3), $\lambda_{c}$ has a bounded second derivative around of $s, 0<b<1$, $n^{1-b} h_{n} \rightarrow 0, n^{b+1} h_{n} \rightarrow \infty, h_{n} \downarrow 0$ as $n \rightarrow \infty$,

a) If $\left(n^{1+b} h_{n}^{5}\right)^{\frac{1}{2}} \rightarrow 0$, then

$$
\left(n^{1+b} h_{n}\right)^{\frac{1}{2}}\left(\hat{\lambda}_{c, n, K}(s)-\lambda_{c}(s)\right) \stackrel{d}{\rightarrow} \operatorname{Normal}\left(0, \sigma^{2}\right)
$$

as $n \rightarrow \infty$, with $\sigma^{2}=\frac{\tau \lambda_{c}(s)}{(1-b)} \int_{-1}^{1} K^{2}(z) d z$.

b) If $\left(n^{1+b} h_{n}^{5}\right)^{\frac{1}{2}} \rightarrow 1$, then

$$
\left(n^{1+b} h_{n}\right)^{\frac{1}{2}}\left(\hat{\lambda}_{c, n, K}(s)-\lambda_{c}(s)\right) \stackrel{d}{\rightarrow} \operatorname{Normal}\left(\mu, \sigma^{2}\right)
$$

as $n \rightarrow \infty$, with $\mu=\frac{\lambda_{c}^{\prime \prime}(s)}{2} \int_{-1}^{1} z^{2} K(z) d z$ and $\sigma^{2}=\frac{\tau \lambda_{c}(s)}{(1-b)} \int_{-1}^{1} K^{2}(z) d z$.

\section{Theorem 2 (The Asymptotic Normal Distribution for $\hat{\lambda}_{c, n, k}(s), b=1$ )}

Suppose that the intensity $\lambda$ satisfies (1) and locally integrable. The kernel function $K$ satisfies (K1), (K2), (K3), $\lambda_{c}$ has a bounded second derivative around of $s, b=1$, $\ln (n) h_{n} \rightarrow 0, \frac{n^{2} h_{n}}{\ln (n)} \rightarrow \infty, h_{n} \downarrow 0$ as $n \rightarrow \infty$,

a) If $\left(\frac{n^{2} h_{n}^{5}}{\ln (n)}\right)^{\frac{1}{2}} \rightarrow 0$, then

$$
\left(\frac{n^{2} h_{n}}{\ln (n)}\right)^{\frac{1}{2}}\left(\hat{\lambda}_{c, n, K}(s)-\lambda_{c}(s)\right) \stackrel{d}{\rightarrow} \operatorname{Normal}\left(0, \sigma^{2}\right)
$$

as $n \rightarrow \infty$, with $\sigma^{2}=\tau \lambda_{c}(s) \int_{-1}^{1} K^{2}(z) d z$.

b) If $\left(\frac{n^{2} h_{n}^{5}}{\ln (n)}\right)^{\frac{1}{2}} \rightarrow 1$, then

$$
\left(\frac{n^{2} h_{n}}{\ln (n)}\right)^{\frac{1}{2}}\left(\hat{\lambda}_{c, n, K}(s)-\lambda_{c}(s)\right) \stackrel{d}{\rightarrow} \operatorname{Normal}\left(\mu, \sigma^{2}\right)
$$

as $n \rightarrow \infty$, with $\mu=\frac{\lambda_{c}^{\prime \prime}(s)}{2} \int_{-1}^{1} z^{2} K(z) d z$ and $\sigma^{2}=\tau \lambda_{c}(s) \int_{-1}^{1} K^{2}(z) d z$.

Theorem 3 (The Asymptotic Normal Distribution for $\hat{\lambda}_{c, n, k}(s), b>1$ )

Suppose that the intensity $\lambda$ satisfies (1) and locally integrable. The kernel function $K$ satisfies (K1), (K2), (K3), and $\lambda_{c}$ has a bounded second derivative around of $s, b>1$, $n^{2} h_{n} \rightarrow \infty, h_{n} \downarrow 0$ as $n \rightarrow \infty$,

a) If $\left(n^{2} h_{n}^{5}\right)^{\frac{1}{2}} \rightarrow 0$, then

$$
\begin{aligned}
& \qquad\left(n^{2} h_{n}\right)^{\frac{1}{2}}\left(\hat{\lambda}_{c, n, K}(s)-\lambda_{c}(s)\right) \stackrel{d}{\rightarrow} \operatorname{Normal}\left(0, \sigma^{2}\right) \\
& \text { as } n \rightarrow \infty \text {, with } \sigma^{2}=\tau^{2-b} \lambda_{c}(s) \zeta(b) \int_{-1}^{1} K^{2}(z) d z, \\
& \text { and } \zeta(b)=\lim _{n \rightarrow \infty}\left(\sum_{k=1}^{\infty} \frac{1}{k^{b}} I(y+s+k \tau \in[0, n])\right) .
\end{aligned}
$$


b) If $\left(n^{2} h_{n}^{5}\right)^{\frac{1}{2}} \rightarrow 1$, then

$$
\left(n^{2} h_{n}\right)^{\frac{1}{2}}\left(\hat{\lambda}_{c, n, K}(s)-\lambda_{c}(s)\right) \stackrel{d}{\rightarrow} \operatorname{Normal}\left(\mu, \sigma^{2}\right)
$$

as $n \rightarrow \infty$, with $\mu=\frac{\lambda_{c}^{\prime \prime}(s)}{2} \int_{-1}^{1} z^{2} K(z) d z, \sigma^{2}=\tau^{2-b} \lambda_{c}(s) \zeta(b) \int_{-1}^{1} K^{2}(z) d z$, and $\zeta(b)=$ $\lim _{n \rightarrow \infty}\left(\sum_{k=1}^{\infty} \frac{1}{k^{b}} I(y+s+k \tau \in[0, n])\right)$.

The proofs of Theorem 1, Theorem 2, and Theorem 3 above can be proved through a rough analysis, [18]. It is recommended to study the basic theory to proof these theorems in [19]-[21].

\section{RESULTS AND DISCUSSION}

Suppose that $\phi$ denotes the standard normal distribution with $\phi^{-1}$ is the inverse. Based on Theorem 1, Theorem 2, and Theorem 3 above, it can be given some confidence interval for $\lambda_{c}$ with significant level $1-\alpha$ as follows:

\section{Corollary 1 (The confidence interval for $\lambda_{c}$ for $0<b<1$ )}

Suppose that all conditions on Theorem 1 are satisfied, the for a significant level $\alpha$ where $0<\alpha<1$, the confidence interval for $\lambda_{c}$ for $0<b<1$ has been given in the following conditions:

a) If $\left(n^{1+b} h_{n}^{5}\right)^{\frac{1}{2}} \rightarrow 0$ then

$$
I_{\lambda_{c}}=\left(\hat{\lambda}_{c, n, k}(s)-\frac{\sigma \phi^{-1}\left(1-\frac{\alpha}{2}\right)}{\sqrt{n^{1+b} h_{n}}}, \hat{\lambda}_{c, n, k}(s)+\frac{\sigma \phi^{-1}\left(1-\frac{\alpha}{2}\right)}{\sqrt{n^{1+b} h_{n}}}\right),
$$

where $\sigma^{2}=\frac{\tau \lambda_{c}(s)}{(1-b)} \int_{-1}^{1} K^{2}(z) d z$.

b) If $\left(n^{1+b} h_{n}^{5}\right)^{\frac{1}{2}} \rightarrow 1$ then

$$
I_{\lambda_{c}}=\left(\hat{\lambda}_{c, n, k}(s)-\frac{\sigma \phi^{-1}\left(1-\frac{\alpha}{2}\right)+\mu}{\sqrt{n^{1+b} h_{n}}}, \hat{\lambda}_{c, n, k}(s)+\frac{\sigma \phi^{-1}\left(1-\frac{\alpha}{2}\right)-\mu}{\sqrt{n^{1+b} h_{n}}}\right),
$$

where $\mu=\frac{\lambda_{c}^{\prime \prime}(s)}{2} \int_{-1}^{1} z^{2} K(z) d z$ and $\sigma^{2}=\frac{\tau \lambda_{c}(s)}{(1-b)} \int_{-1}^{1} K^{2}(z) d z$.

\section{Corollary 2 (The confidence interval for $\lambda_{c}$ for $b=1$ )}

Suppose that all conditions on Theorem 2 are satisfied, the for a significant level $\alpha$ where $0<\alpha<1$, the confidence interval for $\lambda_{c}$ for $b=1$ has been given in the following conditions:

a) If $\left(\frac{n^{2} h_{n}^{5}}{\ln (n)}\right)^{\frac{1}{2}} \rightarrow 0$ then

$$
I_{\lambda_{c}}=\left(\hat{\lambda}_{c, n, k}(s)-\sigma \sqrt{\frac{\ln (n)}{n^{2} h_{n}}} \phi^{-1}\left(1-\frac{\alpha}{2}\right), \hat{\lambda}_{c, n, k}(s)+\sigma \sqrt{\frac{\ln (n)}{n^{2} h_{n}}} \phi^{-1}\left(1-\frac{\alpha}{2}\right)\right),
$$

where $\sigma^{2}=\tau \lambda_{c}(s) \int_{-1}^{1} K^{2}(z) d z$. 
b) If $\left(\frac{n^{2} h_{n}^{5}}{\ln (n)}\right)^{\frac{1}{2}} \rightarrow 1$ then

$I_{\lambda_{c}}=\left(\hat{\lambda}_{c, n, k}(s)-\left(\sigma \phi^{-1}\left(1-\frac{\alpha}{2}\right)+\mu\right) \sqrt{\frac{\ln (n)}{n^{2} h_{n}}}, \hat{\lambda}_{c, n, k}(s)+\left(\sigma \phi^{-1}\left(1-\frac{\alpha}{2}\right)-\mu\right) \sqrt{\frac{\ln (n)}{n^{2} h_{n}}}\right)$,

where $\mu=\frac{\lambda_{c}^{\prime \prime}(s)}{2} \int_{-1}^{1} z^{2} K(z) d z$ and $\sigma^{2}=\tau \lambda_{c}(s) \int_{-1}^{1} K^{2}(z) d z$.

\section{Corollary 3 (The confidence interval for $\lambda_{c}$ for $b>1$ )}

Suppose that all conditions on Theorem 3 are satisfied, the for a significant level $\alpha$ where $0<\alpha<1$, the confidence interval for $\lambda_{c}$ for $b>1$ has been given in the following conditions:

a) If $\left(n^{2} h_{n}^{5}\right)^{\frac{1}{2}} \rightarrow 0$ then

$$
I_{\lambda_{c}}=\left(\hat{\lambda}_{c, n, k}(s)-\frac{\sigma \phi^{-1}\left(1-\frac{\alpha}{2}\right)}{\sqrt{n^{2} h_{n}}}, \hat{\lambda}_{c, n, k}(s)+\frac{\sigma \phi^{-1}\left(1-\frac{\alpha}{2}\right)}{\sqrt{n^{2} h_{n}}}\right),
$$

where $\sigma^{2}=\tau^{2-b} \lambda_{c}(s) \zeta(b) \int_{-1}^{1} K^{2}(z) d z$

b) If $\left(n^{1+b} h_{n}^{5}\right)^{\frac{1}{2}} \rightarrow 1$ then

$$
I_{\lambda_{c}}=\left(\hat{\lambda}_{c, n, k}(s)-\frac{\sigma \phi^{-1}\left(1-\frac{\alpha}{2}\right)+\mu}{\sqrt{n^{2} h_{n}}}, \hat{\lambda}_{c, n, k}(s)+\frac{\sigma \phi^{-1}\left(1-\frac{\alpha}{2}\right)-\mu}{\sqrt{n^{2} h_{n}}}\right),
$$

where $\mu=\frac{\lambda_{c}^{\prime \prime}(s)}{2} \int_{-1}^{1} z^{2} K(z) d z, \sigma^{2}=\tau^{2-b} \lambda_{c}(s) \zeta(b) \int_{-1}^{1} K^{2}(z) d z$, and $\zeta(b)=\lim _{n \rightarrow \infty}\left(\sum_{k=1}^{\infty} \frac{1}{k^{b}} I(y+s+k \tau \in[0, n])\right)$.

To strengthen the reasons for the above confidence intervals, it is given the probability convergence theorems for these confidence interval.

Theorem 4. Convergence in Probability of the Confidence Interval for $\lambda_{c}$ and $0<$ $b<1$

If $\hat{\lambda}_{c, n, k}$ is the estimator for periodic component of the intensity function that is given in equation (3). Also, $I_{\lambda_{c, n}}$ is a confidence interval that is given in Corollary 1 , then for the value $0<\mathrm{b}<1$ satisfies

$$
\mathrm{P}\left(\lambda_{\mathrm{c}, \mathrm{n}}(\mathrm{s}) \in \mathrm{I}_{\lambda_{\mathrm{c}, \mathrm{n}}}\right) \rightarrow 1-\alpha+\mathrm{o}(1)
$$

provided $n \rightarrow \infty$.

\section{The proof of Theorem 4:}

Case (a) Assumption $\left(n^{1+b} h_{n}^{5}\right)^{\frac{1}{2}} \rightarrow 0$

$\mathrm{P}\left(\lambda_{\mathrm{c}}(\mathrm{s}) \in \mathrm{I}_{\lambda_{\mathrm{c}}}\right)=P\left(\hat{\lambda}_{\mathrm{c}, \mathrm{n}, \mathrm{k}}-\frac{\sigma \phi^{-1}\left(1-\frac{\alpha}{2}\right)}{\sqrt{n^{1+b} h_{n}}} \leq \lambda_{\mathrm{c}, \mathrm{n}}(\mathrm{s}) \leq \hat{\lambda}_{\mathrm{c}, \mathrm{n}, \mathrm{k}}+\frac{\sigma \phi^{-1}\left(1-\frac{\alpha}{2}\right)}{\sqrt{n^{1+b} h_{n}}}\right)$ 


$$
\begin{aligned}
& =\mathrm{P}\left(-\frac{\sigma \phi^{-1}\left(1-\frac{\alpha}{2}\right)}{\sqrt{n^{1+b} h_{n}}} \leq \lambda_{\mathrm{c}, \mathrm{n}}(\mathrm{s})-\hat{\lambda}_{\mathrm{c}, \mathrm{n}, \mathrm{k}} \leq \frac{\sigma \phi^{-1}\left(1-\frac{\alpha}{2}\right)}{\sqrt{n^{1+b} h_{n}}}\right) \\
& =P\left(-\frac{\sigma \phi^{-1}\left(1-\frac{\alpha}{2}\right)}{\sqrt{n^{1+b} h_{n}}} \leq \hat{\lambda}_{\mathrm{c}, \mathrm{n}, \mathrm{k}}-\lambda_{\mathrm{c}, \mathrm{n}}(\mathrm{s}) \leq \frac{\sigma \phi^{-1}\left(1-\frac{\alpha}{2}\right)}{\sqrt{n^{1+b} h_{n}}}\right) \\
& =P\left(-\sigma \Phi^{-1}\left(1-\frac{\alpha}{2}\right) \leq \sqrt{n^{1+b} h_{n}}\left(\hat{\lambda}_{c, \mathrm{n}, \mathrm{k}}-\lambda_{\mathrm{c}, \mathrm{n}}(\mathrm{s})\right) \leq \sigma \Phi^{-1}\left(1-\frac{\alpha}{2}\right)\right),
\end{aligned}
$$

Let $Y=\sqrt{n^{1+b} h_{n}}\left(\hat{\lambda}_{c, n, k}-\lambda_{c}(s)\right)$, then based on Theorem $1 Y \sim \operatorname{Normal}\left(0, \sigma^{2}\right)$, By using Central Limit Theorem

Therefore

$$
Z=\frac{Y}{\sigma} \sim \operatorname{Normal}(0,1)
$$

$$
\begin{aligned}
\mathrm{P}\left(\lambda_{\mathrm{c}}(\mathrm{s}) \in \mathrm{I}_{\lambda_{\mathrm{c}}}\right)= & P\left(-\phi^{-1}\left(1-\frac{\alpha}{2}\right) \leq Z \leq \phi^{-1}\left(1-\frac{\alpha}{2}\right)\right) \\
& =\mathrm{P}\left(Z \leq \phi^{-1}\left(1-\frac{\alpha}{2}\right)\right)-P\left(Z<-\phi^{-1}\left(1-\frac{\alpha}{2}\right)\right) .
\end{aligned}
$$

Since the normal distribution has a symmetricity property,

then

$$
P\left(Z<-\phi^{-1}\left(1-\frac{\alpha}{2}\right)\right)=P\left(Z \geq-\phi^{-1}\left(1-\frac{\alpha}{2}\right)\right)
$$

$$
\begin{aligned}
\mathrm{P}\left(\lambda_{\mathrm{c}}(\mathrm{s}) \in \mathrm{I}_{\lambda_{\mathrm{c}}}\right)= & \mathrm{P}\left(Z \leq \phi^{-1}\left(1-\frac{\alpha}{2}\right)\right)-\left(Z \geq-\phi^{-1}\left(1-\frac{\alpha}{2}\right)\right) \\
& =\mathrm{P}\left(Z \leq \phi^{-1}\left(1-\frac{\alpha}{2}\right)\right)-1+\mathrm{P}\left(Z \leq \phi^{-1}\left(1-\frac{\alpha}{2}\right)\right) \\
& =\phi\left(\phi^{-1}\left(1-\frac{\alpha}{2}\right)\right)-1+\phi\left(\phi^{-1}\left(1-\frac{\alpha}{2}\right)\right) \\
& =1-\frac{\alpha}{2}-1+1-\frac{\alpha}{2}=1-\alpha, \text { provided } n \rightarrow \infty .
\end{aligned}
$$

Case (b). Assumption $\left(n^{1+b} h_{n}^{5}\right)^{\frac{1}{2}} \rightarrow 1$

$$
\begin{aligned}
& \mathrm{P}\left(\lambda_{\mathrm{c}}(\mathrm{s}) \in \mathrm{I}_{\lambda_{\mathrm{c}}}\right) \\
& =P\left(\hat{\lambda}_{\mathrm{c}, \mathrm{n}, \mathrm{k}}-\frac{\sigma \phi^{-1}\left(1-\frac{\alpha}{2}\right)+\mu}{\sqrt{n^{1+b} h_{n}}} \leq \lambda_{c, \mathrm{n}}(\mathrm{s}) \leq \hat{\lambda}_{c, \mathrm{n}, \mathrm{k}}+\frac{\sigma \phi^{-1}\left(1-\frac{\alpha}{2}\right)-\mu}{\sqrt{n^{1+b} h_{n}}}\right) \\
& =\mathrm{P}\left(-\frac{\sigma \phi^{-1}\left(1-\frac{\alpha}{2}\right)+\mu}{\sqrt{n^{1+b} h_{n}}} \leq \lambda_{c, \mathrm{n}}(\mathrm{s})-\hat{\lambda}_{c, \mathrm{n}, \mathrm{k}} \leq \frac{\sigma \phi^{-1}\left(1-\frac{\alpha}{2}\right)-\mu}{\sqrt{n^{1+b} h_{n}}}\right) \\
& =P\left(-\frac{\sigma \phi^{-1}\left(1-\frac{\alpha}{2}\right)-\mu}{\sqrt{n^{1+b} h_{n}}} \leq \hat{\lambda}_{c, \mathrm{n}, \mathrm{k}}-\lambda_{c, \mathrm{n}}(\mathrm{s}) \leq \frac{\sigma \phi^{-1}\left(1-\frac{\alpha}{2}\right)+\mu}{\sqrt{n^{1+b} h_{n}}}\right)
\end{aligned}
$$




$$
=P\left(-\left(\sigma \phi^{-1}\left(1-\frac{\alpha}{2}\right)-\mu\right) \leq \sqrt{n^{1+b} h_{n}}\left(\hat{\lambda}_{c, \mathrm{n}, \mathrm{k}}-\lambda_{\mathrm{c}, \mathrm{n}}(\mathrm{s})\right) \leq\left(\sigma \phi^{-1}\left(1-\frac{\alpha}{2}\right)+\mu\right)\right)
$$

Suppose that $Y=\sqrt{n^{1+b} h_{n}}\left(\hat{\lambda}_{c, \mathrm{n}, \mathrm{k}}-\lambda_{\mathrm{c}}(\mathrm{s})\right)$ then based on Theorem 1b $Y \sim \operatorname{Normal}\left(\mu, \sigma^{2}\right)$, and

Therefore

$$
Z=\frac{Y-\mu}{\sigma} \sim \operatorname{Normal}(0,1) .
$$

$$
\begin{aligned}
\mathrm{P}\left(\lambda_{\mathrm{c}}(\mathrm{s}) \in \mathrm{I}_{\lambda_{\mathrm{c}}}\right)= & P\left(-\phi^{-1}\left(1-\frac{\alpha}{2}\right) \leq Z \leq \phi^{-1}\left(1-\frac{\alpha}{2}\right)\right) \\
& =\mathrm{P}\left(Z \leq \phi^{-1}\left(1-\frac{\alpha}{2}\right)\right)-P\left(Z<-\phi^{-1}\left(1-\frac{\alpha}{2}\right)\right) .
\end{aligned}
$$

Since the normal distribution has a symmetricity property

$$
P\left(Z<-\phi^{-1}\left(1-\frac{\alpha}{2}\right)\right)=P\left(Z \geq-\phi^{-1}\left(1-\frac{\alpha}{2}\right)\right),
$$

so

$$
\begin{aligned}
\mathrm{P}\left(\lambda_{\mathrm{c}}(\mathrm{s}) \in \mathrm{I}_{\lambda_{\mathrm{c}}}\right)= & \mathrm{P}\left(Z \leq \phi^{-1}\left(1-\frac{\alpha}{2}\right)\right)-\left(Z \geq-\phi^{-1}\left(1-\frac{\alpha}{2}\right)\right) \\
& =\mathrm{P}\left(Z \leq \phi^{-1}\left(1-\frac{\alpha}{2}\right)\right)-1+\mathrm{P}\left(Z \leq \phi^{-1}\left(1-\frac{\alpha}{2}\right)\right) \\
& =\phi\left(\phi^{-1}\left(1-\frac{\alpha}{2}\right)\right)-1+\phi\left(\phi^{-1}\left(1-\frac{\alpha}{2}\right)\right) \\
& =1-\frac{\alpha}{2}-1+1-\frac{\alpha}{2}=1-\alpha, \text { provided } n \rightarrow \infty .
\end{aligned}
$$

Theorem 5. Convergence in Probability of the Confidence Interval for $\lambda_{c}$ and $b=1$

If $\hat{\lambda}_{c, n, k}$ is the estimator for periodic component of the intensity function that is given in equation (3). Also, $I_{\lambda_{c}}$ is an confidence interval that is given in Corollary 2 , then for the value $b=1$ satisfies

$$
\mathrm{P}\left(\lambda_{\mathrm{c}}(\mathrm{s}) \in \mathrm{I}_{\lambda_{\mathrm{c}}}\right) \rightarrow 1-\alpha+\mathrm{o}(1),
$$

provided $\mathrm{n} \rightarrow \infty$.

\section{The proof of Theorem 5}

Case (a) Assumption $\left(\frac{n^{2} h_{n}^{5}}{\ln (n)}\right)^{\frac{1}{2}} \rightarrow \mathbf{0}$

$\mathrm{P}\left(\lambda_{\mathrm{c}}(\mathrm{s}) \in \mathrm{I}_{\lambda_{\mathrm{c}}}\right)$

$=P\left(\hat{\lambda}_{c, \mathrm{n}, \mathrm{k}}-\sigma \sqrt{\frac{\ln (n)}{n^{2} h_{n}}} \phi^{-1}\left(1-\frac{\alpha}{2}\right) \leq \lambda_{c, \mathrm{n}}(\mathrm{s}) \leq \hat{\lambda}_{\mathrm{c}, \mathrm{n}, \mathrm{k}}+\sigma \sqrt{\frac{\ln (n)}{n^{2} h_{n}}} \phi^{-1}\left(1-\frac{\alpha}{2}\right)\right)$

$=\mathrm{P}\left(-\sigma \sqrt{\frac{\ln (n)}{n^{2} h_{n}}} \phi^{-1}\left(1-\frac{\alpha}{2}\right) \leq \lambda_{c, \mathrm{n}}(\mathrm{s})-\hat{\lambda}_{\mathrm{c}, \mathrm{n}, \mathrm{k}} \leq \sigma \sqrt{\frac{\ln (n)}{n^{2} h_{n}}} \phi^{-1}\left(1-\frac{\alpha}{2}\right)\right)$ 
$=P\left(-\sigma \sqrt{\frac{\ln (n)}{n^{2} h_{n}}} \phi^{-1}\left(1-\frac{\alpha}{2}\right) \leq \hat{\lambda}_{c, \mathrm{n}, \mathrm{k}}-\lambda_{\mathrm{c}, \mathrm{n}}(\mathrm{s}) \leq \sigma \sqrt{\frac{\ln (n)}{n^{2} h_{n}}} \phi^{-1}\left(1-\frac{\alpha}{2}\right)\right)$

$=P\left(-\sigma \phi^{-1}\left(1-\frac{\alpha}{2}\right) \leq \sqrt{\frac{n^{2} h_{n}}{\ln (n)}}\left(\hat{\lambda}_{c, \mathrm{n}, \mathrm{k}}-\lambda_{\mathrm{c}, \mathrm{n}}(\mathrm{s})\right) \leq \sigma \phi^{-1}\left(1-\frac{\alpha}{2}\right)\right)$.

Let $=\sqrt{\frac{n^{2} h_{n}}{\ln (n)}}\left(\hat{\lambda}_{c, n, k}-\lambda_{c}(s)\right)$, then based on Theorem $2 Y \sim \operatorname{Normal}\left(0, \sigma^{2}\right)$, By using Central Limit Theorem

$$
Z=\frac{Y}{\sigma} \sim \operatorname{Normal}(0,1)
$$

Therefore

$$
\mathrm{P}\left(\lambda_{\mathrm{c}}(\mathrm{s}) \in \mathrm{I}_{\lambda_{\mathrm{c}}}\right)=P\left(-\phi^{-1}\left(1-\frac{\alpha}{2}\right) \leq Z \leq \phi^{-1}\left(1-\frac{\alpha}{2}\right)\right)
$$

By using the same arguments before, it is obtained

$$
\mathrm{P}\left(\lambda_{\mathrm{c}}(\mathrm{s}) \in \mathrm{I}_{\lambda_{\mathrm{c}}}\right)=1-\alpha, \text { provided } n \rightarrow \infty .
$$

Case (b). Assumption $\left(\frac{n^{2} h_{n}^{5}}{\ln (n)}\right)^{\frac{1}{2}} \rightarrow \mathbf{1}$

$\mathrm{P}\left(\lambda_{\mathrm{c}}(\mathrm{s}) \in \mathrm{I}_{\lambda_{\mathrm{c}}}\right)$

$=P\left(\hat{\lambda}_{c, \mathrm{n}, \mathrm{k}}-\left(\sigma \phi^{-1}\left(1-\frac{\alpha}{2}\right)+\mu\right) \sqrt{\frac{\ln (n)}{n^{2} h_{n}}} \leq \lambda_{\mathrm{c}}(\mathrm{s}) \leq \hat{\lambda}_{\mathrm{c}, \mathrm{n}, \mathrm{k}}+\left(\sigma \phi^{-1}\left(1-\frac{\alpha}{2}\right)-\mu\right) \sqrt{\frac{\ln (n)}{n^{2} h_{n}}}\right)$

$\left.\left.=\mathrm{P}\left(-\sigma \phi^{-1}\left(1-\frac{\alpha}{2}\right)+\mu\right) \sqrt{\frac{\ln (n)}{n^{2} h_{n}}} \leq \lambda_{c}(\mathrm{~s})-\hat{\lambda}_{c, n, \mathrm{k}} \leq \sigma \phi^{-1}\left(1-\frac{\alpha}{2}\right)-\mu\right) \sqrt{\frac{\ln (n)}{n^{2} h_{n}}}\right)$

$\left.\left.=P\left(-\phi^{-1}\left(1-\frac{\alpha}{2}\right)-\mu\right) \sqrt{\frac{\ln (n)}{n^{2} h_{n}}} \leq \hat{\lambda}_{c, \mathrm{n}, \mathrm{k}}-\lambda_{\mathrm{c}}(\mathrm{s}) \leq \sigma \phi^{-1}\left(1-\frac{\alpha}{2}\right)+\mu\right) \sqrt{\frac{\ln (n)}{n^{2} h_{n}}}\right)$

$=P\left(-\left(\sigma \phi^{-1}\left(1-\frac{\alpha}{2}\right)-\mu\right) \leq \sqrt{\frac{n^{2} h_{n}}{\ln (n)}}\left(\hat{\lambda}_{\mathrm{c}, \mathrm{n}, \mathrm{k}}-\lambda_{\mathrm{c}}(\mathrm{s})\right) \leq\left(\sigma \phi^{-1}\left(1-\frac{\alpha}{2}\right)+\mu\right)\right)$,

Suppose that $Y=\sqrt{\frac{n^{2} h_{n}}{\ln (n)}}\left(\hat{\lambda}_{c, n, \mathrm{k}}-\lambda_{\mathrm{c}}(\mathrm{s})\right)$, then according to Theorem $1 \mathrm{~b}$ $Y \sim \operatorname{Normal}\left(\mu, \sigma^{2}\right)$ and

$$
Z=\frac{Y-\mu}{\sigma} \sim \operatorname{Normal}(0,1) .
$$

Therefore

$\mathrm{P}\left(\lambda_{c, \mathrm{n}}(\mathrm{s}) \in \mathrm{I}_{\lambda_{c, \mathrm{n}}}\right)=P\left(-\phi^{-1}\left(1-\frac{\alpha}{2}\right) \leq Z \leq \phi^{-1}\left(1-\frac{\alpha}{2}\right)\right)$.

The same arguments gave us

$$
\mathrm{P}\left(\lambda_{\mathrm{c}}(\mathrm{s}) \in \mathrm{I}_{\lambda_{\mathrm{c}}}\right)=1-\alpha, \text { provided } n \rightarrow \infty .
$$




\section{Theorem 6. Convergence in Probability of the Confidence Interval for $\lambda_{c, n}$} and $b>1$

If $\hat{\lambda}_{c, n, k}$ is the estimator for periodic component of the intensity function that is given in equation (3). Also, $I_{\lambda_{c, n}}$ is a confidence interval that is given in Corollary 3 , then for the value $b>1$ satisfies

$$
\mathrm{P}\left(\lambda_{\mathrm{c}}(\mathrm{s}) \in \mathrm{I}_{\lambda_{\mathrm{c}}}\right) \rightarrow 1-\alpha+\mathrm{o}(1),
$$

provided $\mathrm{n} \rightarrow \infty$.

\section{The proof of Theorem 6}

Case a. Assumption $\left(n^{2} h_{n}^{5}\right)^{\frac{1}{2}} \rightarrow 0$

$$
\begin{aligned}
& \mathrm{P}\left(\lambda_{\mathrm{c}}(\mathrm{s}) \in \mathrm{I}_{\lambda_{\mathrm{c}}}\right) \\
& =P\left(\hat{\lambda}_{\mathrm{c}, \mathrm{n}, \mathrm{k}}-\frac{\sigma \phi^{-1}\left(1-\frac{\alpha}{2}\right)}{\sqrt{n^{2} h_{n}}} \leq \lambda_{\mathrm{c}}(\mathrm{s}) \leq \hat{\lambda}_{\mathrm{c}, \mathrm{n}, \mathrm{k}}+\frac{\sigma \phi^{-1}\left(1-\frac{\alpha}{2}\right)}{\sqrt{n^{2} h_{n}}}\right) \\
& =\mathrm{P}\left(-\frac{\sigma \phi^{-1}\left(1-\frac{\alpha}{2}\right)}{\sqrt{n^{2} h_{n}}} \leq \lambda_{\mathrm{c}}(\mathrm{s})-\hat{\lambda}_{\mathrm{c}, \mathrm{n}, \mathrm{k}} \leq \frac{\sigma \phi^{-1}\left(1-\frac{\alpha}{2}\right)}{\sqrt{n^{2} h_{n}}}\right) \\
& =P\left(-\sigma \frac{\sigma \phi^{-1}\left(1-\frac{\alpha}{2}\right)}{\sqrt{n^{2} h_{n}}} \leq \hat{\lambda}_{\mathrm{c}, \mathrm{n}, \mathrm{k}}-\lambda_{\mathrm{c}}(\mathrm{s}) \leq \sigma \frac{\sigma \phi^{-1}\left(1-\frac{\alpha}{2}\right)}{\sqrt{n^{2} h_{n}}}\right) \\
& =P\left(-\sigma \phi^{-1}\left(1-\frac{\alpha}{2}\right) \leq \sqrt{n^{2} h_{n}}\left(\hat{\lambda}_{\mathrm{c}, \mathrm{n}, \mathrm{k}}-\lambda_{\mathrm{c}}(\mathrm{s})\right) \leq \sigma \phi^{-1}\left(1-\frac{\alpha}{2}\right)\right) .
\end{aligned}
$$

Let $Y=\sqrt{n^{2} h_{n}}\left(\hat{\lambda}_{\mathrm{c}, \mathrm{n}, \mathrm{k}}-\lambda_{\mathrm{c}}(\mathrm{s})\right.$, then according to Theorem $3 \mathrm{a} Y \sim \operatorname{Normal}\left(0, \sigma^{2}\right)$ and

$$
Z=\frac{Y}{\sigma} \sim \operatorname{Normal}(0,1)
$$

Therefore

$$
\mathrm{P}\left(\lambda_{\mathrm{c}}(\mathrm{s}) \in \mathrm{I}_{\lambda_{\mathrm{c}}}\right)=P\left(-\phi^{-1}\left(1-\frac{\alpha}{2}\right) \leq Z \leq \phi^{-1}\left(1-\frac{\alpha}{2}\right)\right) .
$$

The same arguments gave us

$$
\mathrm{P}\left(\lambda_{\mathrm{c}}(\mathrm{s}) \in \mathrm{I}_{\lambda_{\mathrm{c}}}\right)=1-\alpha, \text { provided } n \rightarrow \infty .
$$

Case b. Assumption $\left(n^{2} h_{n}^{5}\right)^{\frac{1}{2}} \rightarrow \mathbf{1}$

$$
\begin{aligned}
& \mathrm{P}\left(\lambda_{\mathrm{c}}(\mathrm{s}) \in \mathrm{I}_{\lambda_{\mathrm{c}}}\right) \\
& =P\left(\hat{\lambda}_{\mathrm{c}, \mathrm{n}, \mathrm{k}}-\frac{\sigma \phi^{-1}\left(1-\frac{\alpha}{2}\right)+\mu}{\sqrt{n^{2} h_{n}}} \leq \lambda_{\mathrm{c}}(\mathrm{s}) \leq \hat{\lambda}_{\mathrm{c}, \mathrm{n}, \mathrm{k}}+\frac{\sigma \phi^{-1}\left(1-\frac{\alpha}{2}\right)-\mu}{\sqrt{n^{2} h_{n}}}\right) \\
& =\mathrm{P}\left(-\frac{\sigma \phi^{-1}\left(1-\frac{\alpha}{2}\right)+\mu}{\sqrt{n^{2} h_{n}}} \leq \lambda_{\mathrm{c}}(\mathrm{s})-\hat{\lambda}_{\mathrm{c}, \mathrm{n}, \mathrm{k}} \leq \sigma \frac{\sigma \phi^{-1}\left(1-\frac{\alpha}{2}\right)-\mu}{\sqrt{n^{2} h_{n}}}\right)
\end{aligned}
$$




$$
\begin{aligned}
& =P\left(-\frac{\sigma \phi^{-1}\left(1-\frac{\alpha}{2}\right)-\mu}{\sqrt{n^{2} h_{n}}} \leq \hat{\lambda}_{\mathrm{c}, \mathrm{n}, \mathrm{k}}-\lambda_{\mathrm{c}}(\mathrm{s}) \leq \frac{\sigma \phi^{-1}\left(1-\frac{\alpha}{2}\right)+\mu}{\sqrt{n^{2} h_{n}}}\right) \\
& =P\left(-\left(\sigma \phi^{-1}\left(1-\frac{\alpha}{2}\right)-\mu\right) \leq \sqrt{n^{2} h_{n}}\left(\hat{\lambda}_{c, \mathrm{n}, \mathrm{k}}-\lambda_{\mathrm{c}}(\mathrm{s})\right) \leq\left(\sigma \phi^{-1}\left(1-\frac{\alpha}{2}\right)+\mu\right)\right) .
\end{aligned}
$$

Suppose that $Y=\sqrt{n^{2} h_{n}}\left(\hat{\lambda}_{c, n, k}-\lambda_{c}(s)\right.$, then based on Theorem 3b $Y \sim \operatorname{Normal}\left(\mu, \sigma^{2}\right)$ and

$$
Z=\frac{Y-\mu}{\sigma} \sim \operatorname{Normal}(0,1) .
$$

Therefore

$$
\mathrm{P}\left(\lambda_{\mathrm{c}}(\mathrm{s}) \in \mathrm{I}_{\lambda_{\mathrm{c}}}\right)=P\left(-\phi^{-1}\left(1-\frac{\alpha}{2}\right) \leq Z \leq \phi^{-1}\left(1-\frac{\alpha}{2}\right)\right) \text {. }
$$

By using the same arguments, it is obtained that

$$
\mathrm{P}\left(\lambda_{\mathrm{c}}(\mathrm{s}) \in \mathrm{I}_{\lambda_{\mathrm{c}}}\right)=1-\alpha \text {, provided } n \rightarrow \infty .
$$

\section{CONCLUSIONS}

From the results that have been studied, the formula to determine the confidence interval for parameter of the periodic component of the nonhomogeneous Poisson process with the intensity in the form of periodic function has been obtained. These confidence intervals have been given for each case of the values of $b$, this is because the results of previous studies show that the variance of the estimator is given in a different function for each case of the values of $b$. These confidence intervals have been proved to converge in probability $1-\alpha$.

The recommendation for further research that can be done is providing numerical simulations for each confidence interval case, there are 6 cases. The simulation can be started by determining the bandwidth function $h_{n}$ which satisfies all the conditions in the given case and determining the probability of the estimator being in the confidence interval.

\section{REFERENCES}

[1] S. Ghahramani, Fundamentals of Probability: With Stochastic Processes, Third Edition. New Jersey: Pearson Prentice Hall, 2005.

[2] D. J. Daley and D. Vere-Jones, "Basic properties of the Poisson process," An Introd. to Theory Point Process. Vol. I Elem. Theory Methods, pp. 19-40, 2003.

[3] G. Last and M. Penrose, Lectures on the Poisson Process (Vol. 7). Cambridge University Press, 2017.

[4] J. Geng, W. Shi, and G. Hu, "Bayesian nonparametric nonhomogeneous poisson process with applications to usgs earthquake data," arXiv Prepr. arXiv1907.03186, 2019.

[5] F. Grabski, "Nonhomogeneous Poisson process and compound Poisson process in the modelling of random processes related to road accidents," J. KONES, vol. 26, no. 1, pp. 39-46, 2019.

[6] E. Lawrence, S. Vander Wiel, C. Law, S. B. Spolaor, and G. C. Bower, "The nonhomogeneous poisson process for fast radio burst rates," Astron. J., vol. 154, 
[7] R. Helmers and I. W. Mangku, "Estimating the intensity of a cyclic Poisson process in the presence of linear trend," Ann. Inst. Stat. Math., vol. 61, no. 3, pp. 599-628, 2009.

[8] I. W. Mangku, "Estimating the intensity obtained as the product of a periodic function with the linear trend of a non-homogeneous Poisson process," Far East J. Math. Sci., vol. 51, pp. 141-150, 2011.

[9] W. Ismayulia, I. W. Mangku, and S. Siswandi, "Pendugaan komponen periodik fungsi intensitas berbentuk fungsi periodik kali tren linear suatu proses Poisson non-homogen," J. Math. Its Appl., vol. 12, no. 1, pp. 49-62, 2013.

[10] I. W. Mangku, R. Budiarti, Taslim, and Casman, "Estimating the intensity obtained as the product of a periodic function with the quadratic trend of a nonhomogeneous Poisson process," Far East J. Math. Sci., vol. 82, no. 1, pp. 33-44, 2013.

[11] I. W. Mangku, Siswadi, and R. Budiarti, "Consistency of a kernel-type estimator of the intensity of the cyclic Poisson process with the linear trend," J. Indones. Math. Soc., vol. 15, no. 1, pp. 37-48, 2009.

[12] R. Helmers and I. W. Mangku, "Predicting a cyclic Poisson process," Ann. Inst. Stat. Math., vol. 64, no. 6, pp. 1261-1279, 2012.

[13] N. Leonenko, E. Scalas, and M. Trinh, "The fractional non-homogeneous Poisson process," Stat. Probab. Lett., vol. 120, pp. 147-156, 2017.

[14] W. Erliana, "Pendugaan Tipe Kernel Umum untuk Intensitas Berupa Fungsi Periodik Kali Tren Fungsi Pangkat Proses Poisson Nonhomogen," Institut Pertanian Bogor, 2014.

[15] I. Maulidi, I. W. Mangku, and H. Sumarno, "Strong consistency of kernel-type estimator for the intensity obtained as the product of a periodic function with the power function trend of non-homogeneous poisson process," Br. J. Appl. Sci. Technol., vol. 9, no. 4, pp. 383-387, 2015.

[16] I. Maulidi, M. Ihsan, and V. Apriliani, "The numerical simulation for asymptotic normality of the intensity obtained as a product of a periodic function with the power trend function of a nonhomogeneous Poisson process," Desimal J. Mat., vol. 3, no. 3, pp. 271-278, 2020.

[17] R. Helmers, I. W. Mangku, and R. Zitikis, "Consistent estimation of the intensity function of a cyclic Poisson process," J. Multivar. Anal., vol. 84, no. 1, pp. 19-39, 2003.

[18] N. Valentika, I. W. Mangku, and W. Erliana, "Strong consistency and asymptotic distribution of estimator for the intensity function having form of periodic function multiplied by power function trend of a Poisson process," Int. J. Eng. Manag. Res., vol. 8, no. 2, pp. 232-236, 2018.

[19] R. J. Serfling, Approximation Theorems of Mathematical Statistics. John Wiley \& Sons, 1980.

[20] R. V. Hogg, J. McKean, and A. T. Craig, Introduction to Mathematical Statistics (6th Edition). Pearson Education, 2005.

[21] R. M. Dudley, Real Analysis and Probability. Wardswort \& Brooks, 1989. 